\title{
Research on battery peak power control method based on Improved PSO algorithm
}

\author{
Jialong Song ${ }^{1 *}$, Peng Xie ${ }^{1}$ \\ ${ }^{1}$ Zhuhai College of Jilin University, Zhuhai, Guangdong, 519041, China
}

\begin{abstract}
In order to better guarantee the operation effect of power equipment, a battery discharge peak power control method based on Improved PSO algorithm is proposed. The operation parameters between the peak power of battery discharge and external power supply are collected, and the operation parameters are switched by using the support bus. The power acquisition process of battery operation is standardized, and the power of battery power conversion unit is controlled by improving PSO algorithm. The results show that the control error of the battery peak power control method based on the improved PSO algorithm is significantly lower in the actual process, and the control accuracy is improved.
\end{abstract}

\section{Introduction}

With the continuous improvement of power supply reliability requirements in communication, network, industry and other fields, higher requirements are put forward for the charging and discharging control accuracy of ups ${ }^{[1,2]}$. For this reason, a battery discharge peak power control method based on Improved PSO algorithm is proposed. The battery can not only convert the energy between the battery cells, but also keep the power supply constant. Battery power conversion device needs to manage battery charge and discharge. Because the battery is very sensitive to voltage and current, overcharge or over discharge will cause irreparable damage to the battery. Through the estimation of the maximum charging and discharging power of the battery, the charging and discharging capacity of the battery in the limit state can be estimated, so as to avoid overcharge and discharge of the battery and ensure the normal use of the battery. Therefore, the improved PSO algorithm is of great significance for the safe operation of batteries.

\section{Peak power control method of battery discharge}

\section{1 collection of battery discharge peak value}

The hourly discharge rate of the battery is expressed by the basic discharge time, that is, the power supply time of the electronic quantity put into the rated voltage. The standard discharge rate of the battery pack can be expressed as follows:

$$
u_{e}=\prod \mathrm{e}^{*} \frac{Q_{w}+z}{i_{w}^{*} m}
$$

In the above algorithm, I is the standard discharge time rate in hours, $Q_{w}$ is the battery, a (hour) is the unit, ah is the unit, $\mathrm{Z}$ is the unit, and a (ampere) is the unit. When the battery pack reaches a certain capacity within the calibration time, the current value required for positive and negative discharge of the battery pack is the ratio of the discharge current of the battery pack to its rated capacity ${ }^{[3-5]}$. The relationship between the discharge rate and the time discharge rate is multiple in a certain range. The discharge rate of standard battery is described by BP neural network.

$$
A=\sum \lim _{0 \rightarrow \infty} \frac{u_{e} \cdot p+r}{k_{s}+m}
$$

In the above algorithm, $k_{s}$ is the accurate discharge capacity required for the rated discharge of the battery, and $p$ is the rated discharge capacity of the battery. The electrolyzer is composed of solution battery and electrolyzer ${ }^{[6]}$. When the battery pack is working, the electrolyte is injected from the electrolyte, and the positive and negative solutions in the battery pack are separated by the ion membrane. The cation in the electrolyte is filled by an ion-exchange membrane, and an electric current is generated by directional movement. The power supply state of the battery refers to the maximum power that the battery can reach in the current state.

*E-mail: sj1@jluzh.edu.cn 


\subsection{Battery power calculation}

When the continuous discharge power is constant, the terminal voltage decreases to the minimum allowable working voltage, that is, the battery terminal power, which depends on the limiting conditions of battery voltage. In view of the fact that the battery industry generally uses $10 \mathrm{~s}$ power as its maximum power measurement standard, this paper uses $\mathrm{X}$ to represent the number of electrons in the redox reaction of the battery industry electrode, $t_{1}$ represents the redox reaction time, $t_{2}$ represents the reduction reaction time. The accurate parameters can be expressed by the battery life prediction network as follows

$$
e=\frac{1}{2 A \Delta x} \sum_{t_{1}=0}^{\infty} \sum_{t_{2}}^{\infty}(|y-d|)^{2}
$$

$y$ and $d$ represent the remaining life parameters of positive and negative electrode materials respectively. The two-dimensional peak power method is used to denoise the discharge area; the background and the peak value of the brown and white area of the battery abnormal edge area are extracted to remove the redundant data; PSO-RBFNN algorithm and threshold segmentation algorithm are used to judge the background brightness of the abnormal edge area of the battery, and the difference calculation method of the background brightness is given.

(1) The collected abnormal peak power is converted into local value, which is recorded as: $l_{i}{ }^{\prime}$;

(2) The abnormal peak power data are counted, and the background peak power value of each row and column is obtained, and the corresponding number of rows and columns is recorded $L_{i}=\left(l_{1}, l_{2}, \ldots l_{k}\right), H_{j}=\left(h_{1}, h_{2}, \ldots h_{n}\right)$. In the above set, $L_{i}$ represents the gray value of the i-th feature point between rows on the battery surface, and $H_{j}$

represents the gray value of the $\mathrm{j}$-th feature point between columns on the battery surface.

(3) According to step (2), further calculate the threshold value of the abnormal area. By collecting the characteristics of the abnormal area and boundary on the surface of the cell, the maximum immunity of the white background area between the rows and rows of the cell surface is calculated

$$
\begin{gathered}
H\left(x_{\mathrm{i}}, y_{i}\right)=\left\{\begin{array}{l}
L_{i}(x, y), L_{i} \leqslant \text { mean }(L), i \in(1, \mathrm{k}) \\
\text { delete, } \quad \text { otherwise }
\end{array}\right. \\
L\left(x_{j}, y_{\mathrm{j}}\right)= \begin{cases}H_{j}(x, y), H_{j} \leqslant \text { mean }(H), j \in(1, \mathrm{n}) \\
\text { delete, } & \text { otherwise }\end{cases}
\end{gathered}
$$

In the formula, the flow characteristic coordinates are $(\mathrm{x}, \mathrm{y})$, e is the original peak power collected, and its power size is $\mathrm{k}^{*} \mathrm{~g}$, then the color value of the original peak power is extracted. In the process of regional noise anti-noise processing, $\mathrm{m} * \mathrm{n}$ is used. In this environment, the following algorithm is used:

$$
\begin{gathered}
d=E\left(m^{*} n\right) / \operatorname{floorH}\left(x_{\mathrm{i}}, y_{i}\right) \\
f=E\left(k^{*} g\right) / \operatorname{floorL}\left(x_{\mathrm{j}}, y_{j}\right)
\end{gathered}
$$

Through the combination of median filter and particle swarm optimization RBFNN, the peak power noise is further removed. In order to eliminate the noise, it is necessary to classify the one-dimensional and two-dimensional median filters to eliminate the interference of edge details in different regions within the peak power range. Because discharge filter has many characteristics, it is necessary to standardize peak power resolution according to the former algorithm. In this environment, the noise reduction is more direct

$$
g(x, y)=\operatorname{Med}: d^{*}[f(t-x, r-y)-1]
$$

Among them, $\mathrm{G}(\mathrm{x}, \mathrm{y})$ is the maximum power de-noising area, $\mathrm{W}$ is the odd power point generated by denoising, and Med is the median standard value of filtering. This method can effectively obtain the peak power of the battery and suppress the noise. In this paper, a method of electromagnetic wave propagation around dielectric is introduced. Based on the analysis of experimental data, a control method based on Maxwell's classical electromagnetic field equation is proposed. The distance between the edge line and the upper and lower end of the electrode is set as $\mathrm{h} 1$ and $\mathrm{h} 2$ respectively. The specific algorithm is as follows:

$$
\Delta G_{\mu}=\sum \frac{\varpi-1}{2 g(x+y) \sigma\left(\mathrm{h}_{1}+\mathrm{h}_{2}\right)}
$$

The numerical solution of the frequency equation of S-type discharge peak electromagnetic wave is obtained by further rotation angle transformation

$$
\Delta S_{\mu}=\sum \frac{\Delta G_{\mu}-1}{2(\sigma-\varpi)\left(h_{1}-h_{2}\right)}
$$

Combined with the method proposed in this paper, the peak power of battery discharge is further optimized. The detection technology of peak discharge power after noise reduction is studied and analyzed. The non grid detection combined with the characteristics of battery surface structure material can correct the abnormal energy area of battery surface. After noise reduction, the peak power of the battery surface is converted into the peak power of the characteristic value $\mathrm{g}(\mathrm{x}, \mathrm{y})$, and then the measured peak power is detected. Light intensity $\mathrm{P}$ and luminous flux $\mathrm{Q}$ are used to calculate the illuminance and illuminance projection of the battery surface.

$$
W_{i}=\sum_{p=1}^{f} g(x, y)-f
$$




$$
P_{i}=\sum_{\mathrm{q}=1}^{f} g(x, y)-d
$$

According to the calculated value, the power smoothness of the spatial region is determined, and the resolution of the region background neighborhood is improved by using the frequency domain principle. When the peak power is detected by battery discharge, the Fourier parameters of peak power need to be transformed to obtain the spatial component value in frequency domain and spatial filtering. According to the principle of spatial linear filter detection, the battery energy conversion power $m$, battery abnormal area radius $R$, illumination angle $\alpha_{1}$ and $\alpha_{2}$ were standardized, and the unbalanced value of battery energy conversion under constraint conditions was calculated. The specific algorithm is as follows:

$$
\mathrm{E}=2 m R\left|\cos \frac{\alpha_{2}-\alpha_{1}}{2}\right|\left(W_{\mathrm{i}}-P_{i}\right)^{\frac{\alpha_{2}+\alpha_{1}}{2}}
$$

The above algorithm can effectively calculate the electric differential of the battery surface. Due to the irregularity of the battery pack, it is necessary to compare the edge number of peak power between the detector and the detail area, and analyze the grey curve by marking and identifying the difference of peak power, so as to realize the accurate detection of the peak power of the battery pack.

\subsection{Realization of battery power control}

The network structure of this system is five layers. For the same level network, each node has similar functions. O1, I, etc. are the outputs of the first layer I node. Level 1: the nodes in this layer are adaptive nodes, which are represented by node functions. The function is to determine that the given input satisfies the corresponding fuzzy set. First level: the second level node uses node function to represent the adaptive node. Its function is to determine that the given input satisfies the corresponding fuzzy set. The control module mainly completes data acquisition, external communication and other software functions. Then the signal is converted into digital signal through 485 communication, and finally displayed on the host computer.

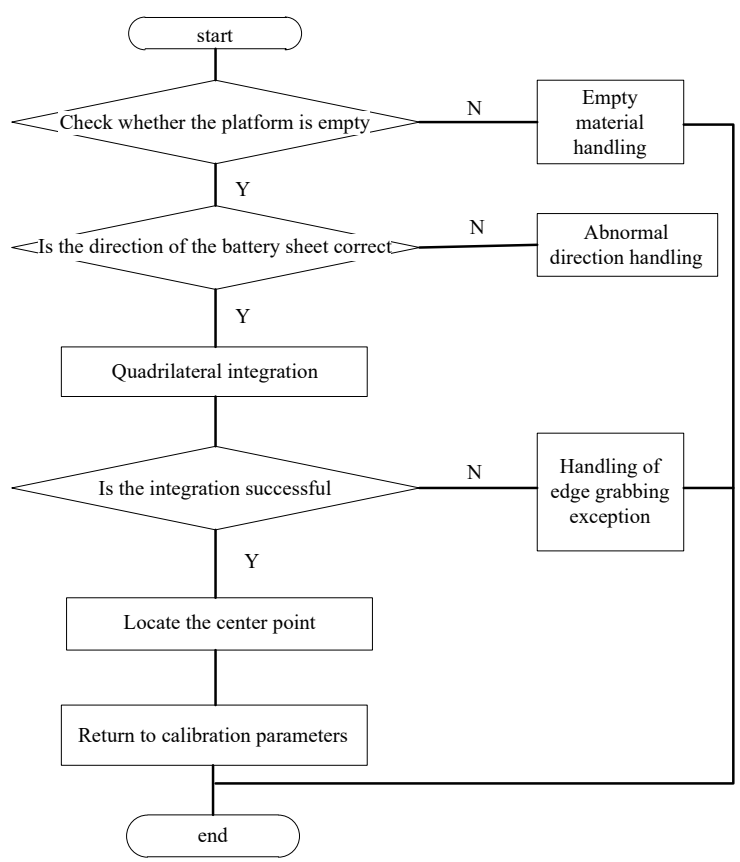

Fig. 1 battery power control steps

Based on the above steps, the peak parameters of battery discharge state are effectively collected and input. According to the information in Table 1, the abnormal power value is reasonably controlled and corrected to ensure the safety and effectiveness of battery operation.

\section{Analysis of experimental results}

In order to verify the effect of this method, the experimental platform is mainly used for the performance test and algorithm verification of battery module, including the battery module to be tested, the main power module, Digatron battery test system, monitoring PC module, high and low temperature box, data acquisition and recording module. During the test, the battery module is placed in the high and low temperature box, and the temperature in the box is adjusted. After waiting for 5 hours, the battery module is consistent with the ambient temperature, and the relevant tests can be started. The monitoring PC carries out various tests on the main power supply startup of the battery module through software settings, and the data acquisition and recording module records the test information such as voltage and current. 12 battery modules and 8 ah lithium manganese dioxide batteries were connected in series, and the data recording time interval was $50 \mathrm{~ms}$. The battery test system can carry out charge and discharge tests under constant power, constant voltage, constant current and other conditions. The response time of the test equipment is $50 \mathrm{~ms}$. The mathematical model of energy device and peak discharge is established by Matlab / Simulink, and verified by simulation. The energy storage system consists of 10 sets of $4 \mathrm{MW}$ fans and batteries. The discharge depth of $8 \mathrm{MW}$ and $14 \mathrm{MW} / \mathrm{h}$ are $10 \% \sim 90 \%$ respectively. DC / AC bidirectional converter is used to discharge the battery pack, and its output peak power is 
controlled by external power supply, internal current and double loop. When $120 \mathrm{~s}$ is selected, not only the smoothing effect of the initial peak power, but also the control requirements after the change of time constant should be considered. Then, the peak flow data collected by the two methods are compared, as shown in the following figure:

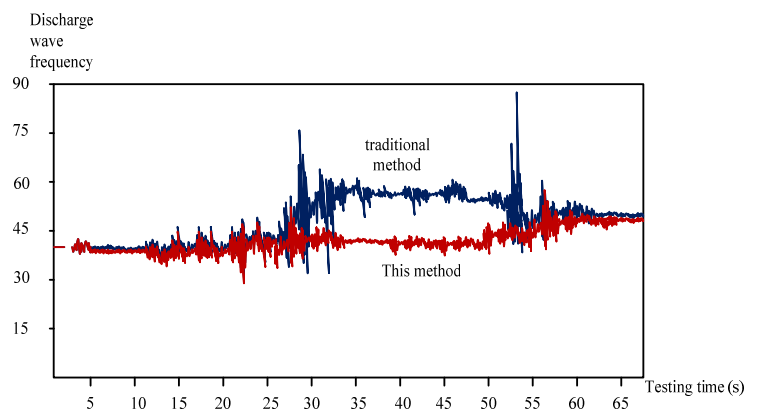

Fig. 2 collection of battery discharge peak value

In order to check whether the battery discharge peak power control method based on the improved PSO algorithm meets the design requirements, 12 battery voltage acquisition ports are connected, and the charging time of each battery is measured randomly in the process of constant voltage charging. The average control data of the two control methods are recorded, and the specific test results are as follows:

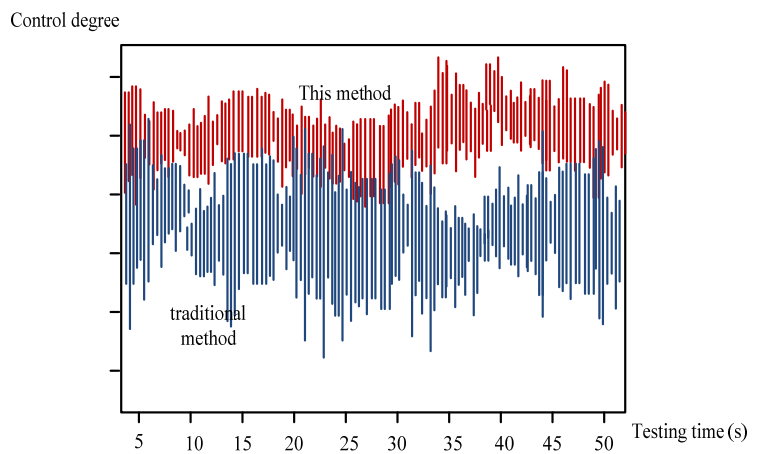

Fig. 3 comparison test results

Compared with the traditional control method, the control error of the battery discharge peak power control method based on the improved PSO algorithm is obviously lower in the actual application process, and the control effect of the battery discharge power is better, which fully meets the research requirements.

\section{Conclusion}

The charging state, voltage and current characteristics of the battery pack were studied, and the battery model was established. The charging and discharging power of the battery is adjusted by adjusting the filtering time constant, and the discharging state of the battery is optimized by fuzzy adjusting the charging and discharging power of the battery. The fluctuation of battery charging (discharging) state and peak discharge power were studied. The experimental results show that the proposed control strategy can effectively balance the peak discharge power and the fluctuation of battery discharge state, and achieve the optimal battery charging state.

\section{Acknowledgments}

This work was supported by Guangdong Province Key Platforms and Major Research Projects(No. 2018KQNCX347): Preparation and Modification Positive Materials (NCM523) of Lithium-ion Battery and Electrochemical Properties Study, Innovative Ability Training Project: Preparation and application of silicon / carbon anode materials for lithium ion batteries (2018XJCQ067), and the Quality engineering projects of Zhuhai College of Jilin University: Guangdong Yutian Bali Technology Co., Ltd. practice teaching base (ZLGC20190306).

\section{References}

1. Fang J, Feng J. Using PSO-TVAC to improve the performance of DV-Hop[J]. International Journal of Wireless and Mobile Computing, 2018, 14(4):358.

2. Anand A, Suganthi L. Hybrid GA-PSO Optimization of Artificial Neural Network for Forecasting Electricity Demand[J]. Energies, 2018, 11(4):728-730.

3. Hu Y, Li J, Hong M, et al. Short term electric load forecasting model and its verification for process industrial enterprises based on hybrid GA-PSO-BPNN algorithm-A case study of papermaking process[J]. Energy, 2019, 170(12):1215-1227.

4. Jannesar M R, Sedighi A, Savaghebi M, et al. Optimal placement, sizing, and daily charge/discharge of battery energy storage in low voltage distribution network with high photovoltaic penetration $[\mathrm{J}]$. Applied Energy, 2018, 226(15):957-966.

5. Yigeng $\mathrm{H}$, Jiani $\mathrm{X}$, Dongdong $\mathrm{Z}$, et al. A Novel Battery State of Charge Estimation Method Based on a Super-Twisting Sliding Mode Observer[J]. Energies, 2018, 11(5):1211.

6. Ye $\mathrm{P}$, Dong $\mathrm{H}, \mathrm{Xu} \mathrm{Y}$, et al. NiCo2O4 surface coating Li[Ni0.03Mn1.97]O4 micro-/nano- spheres as cathode material for high-performance lithium ion battery[J]. Applied Surface ence, 2018, 428(15):469-477. 